\title{
There is No McLaughlin Geometry
}

\author{
Patric R. J. Östergård \\ Department of Communications and Networking \\ Aalto University School of Electrical Engineering \\ P.O. Box 13000, 00076 Aalto, Finland \\ patric.ostergard@aalto.fi \\ Leonard H. Soicher \\ School of Mathematical Sciences \\ Queen Mary University of London \\ Mile End Road \\ London E1 4NS, UK \\ 1.h.soicher@qmul.ac.uk
}

\begin{abstract}
We determine that there is no partial geometry $\mathcal{G}$ with parameters $(s, t, \alpha)=(4,27,2)$. The existence of such a geometry has been a challenging open problem of interest to researchers for almost 40 years. The particular interest in $\mathcal{G}$ is due to the fact that it would have the exceptional McLaughlin graph as its point graph. Our proof makes extensive use of symmetry and high-performance distributed computing, and details of our techniques and checks are provided. One outcome of our work is to show that a pseudogeometric strongly regular graph achieving equality in the Krein bound need not be the point graph of any partial geometry.
\end{abstract}

Keywords: partial geometry; pseudogeometric graph; McLaughlin geometry; McLaughlin graph; Krein bound; backtrack search.

MSC Codes: 51E14 (Primary); 05-04 (Secondary). 


\section{Introduction}

For positive integers $s, t, \alpha$, a partial geometry with parameters $(s, t, \alpha)$, or a $\operatorname{pg}(s, t, \alpha)$, is a point-line incidence structure such that

1. there are exactly $s+1$ points on each line;

2. there are exactly $t+1$ lines through each point;

3. every pair of distinct points are on at most one line (and so every pair of distinct lines meet in at most one point); and

4. for every line $L$ and every point $p$ not on $L$, there are exactly $\alpha$ lines through $p$ meeting $L$.

Omitting the last condition, we obtain the definition of a partial linear space with parameters $(s, t)$, or a $\operatorname{pls}(s, t)$.

Partial geometries, introduced by Bose [1], generalise certain important classes of finite geometries: generalised quadrangles, 2- $(v, s+1,1)$ designs, nets, and the duals of these geometries. See [5] and [27] for useful surveys on partial geometries.

The point graph of a partial linear space $\mathcal{P}$ has the points of $\mathcal{P}$ as vertices and has $\{p, q\}$ as an edge if and only if the points $p$ and $q$ are distinct and on a common line of $\mathcal{P}$. It is well known (and first proved in [1]) that a $\operatorname{pg}(s, t, \alpha)$ has a strongly regular point graph, with parameters

$$
((s+1)(s t+\alpha) / \alpha, s(t+1), s-1+t(\alpha-1),(t+1) \alpha) .
$$

A strongly regular graph with parameters of the form (11), with $s, t, \alpha \in \mathbb{Z}$, $1 \leq s, t$ and $1 \leq \alpha \leq \min \{s+1, t+1\}$, is called a pseudogeometric $(s, t, \alpha)$ graph, and such a graph is called geometric if it is the point graph of at least one $\operatorname{pg}(s, t, \alpha)$. It is known that if a pseudogeometric $(s, t, \alpha)$-graph $\Delta$ is the point graph of a $\operatorname{pls}(s, t)$, then this $\operatorname{pls}(s, t)$ must be a $\operatorname{pg}(s, t, \alpha)$ and $\Delta$ is geometric. This follows from the fact that if a pseudogeometric $(s, t, \alpha)$-graph has an $(s+1)$-clique $C$, then every vertex not in $C$ is adjacent to exactly $\alpha$ vertices in $C$ (see [8, Lemma 1] and its proof).

It has long been an open problem of considerable interest whether there exists a partial geometry $\mathcal{G}$ with parameters $(s, t, \alpha)=(4,27,2)$ (see [4, [5, 15, 17, 25]). Such a geometry would have 275 points and 1540 lines, and the point graph $\Gamma$ of $\mathcal{G}$ would be a strongly regular graph with parameters

$$
(v, k, \lambda, \mu)=(275,112,30,56) \text {. }
$$


Goethals and Seidel [7] showed that such a strongly regular graph is unique (up to isomorphism). Thus $\Gamma$ would be the well-studied and fascinating McLaughlin graph, defined by J. McLaughlin [19] for the construction of his sporadic simple group, now called the McLaughlin group $M c L$. The full automorphism group of $\Gamma$ is $G \cong M c L: 2$, which acts transitively with permutation rank 3 on the set of vertices [19].

As in [25], we call a $\operatorname{pg}(4,27,2)$ a McLaughlin geometry. The main purpose of this paper is finally to settle the existence of a McLaughlin geometry, unfortunately in the negative. We do this by proving that the McLaughlin graph is not the point graph of any $\operatorname{pls}(4,27)$.

The McLaughlin graph $\Gamma$ is an example of a pseudogeometric $(s, t, \alpha)$ graph achieving equality in the Krein bound

$$
(s+1-2 \alpha) t \leq(s-1)(s+1-\alpha)^{2}
$$

(see [4, Theorem 7.6]). The problem of the existence of a McLaughlin geometry appears first to have been posed in [4], and was in connection with [4, Question 7.10] asking whether every pseudogeometric $(s, t, \alpha)$-graph achieving equality in the Krein bound is geometric. It was proved in [4, Theorem 7.9] that this is indeed the case when $\alpha=1$, so the McLaughlin graph was a particularly important test case. See also the Remark and Open Question in [5, p. 442]. The nonexistence of a McLaughlin geometry now shows that a pseudogeometric $(s, t, \alpha)$-graph achieving equality in the Krein bound need not be geometric when $\alpha>1$.

For brevity, throughout this paper we omit the term putative when writing about putative McLaughlin geometries and their substructures. For our proof, we fix a copy $\Gamma$ of the McLaughlin graph. In the Appendix, we give explicit permutation generators for the automorphism group $G$ of $\Gamma$, together with the construction of $\Gamma$ from $G$. Although we focus on one specific pseudogeometric graph, the main ideas and techniques discussed are applicable more generally.

\section{Candidate lines of a McLaughlin geometry}

Given our copy $\Gamma$ of the McLaughlin graph, the first step is to determine the candidates for lines of a McLaughlin geometry. Each candidate line is a 5 -clique in $\Gamma$. There are exactly 154005 -cliques in $\Gamma$, which can be readily obtained using Cliquer [20] or GRAPE [26] (see, for example, the Appendix). We denote the set of these 15400 cliques (or candidate lines, or simply lines) by $\mathcal{C}$. 
We now give some basic properties of these cliques, which can also be easily checked computationally.

Lemma 1. 1. Each vertex in $\Gamma$ is in exactly 280 of the cliques in $\mathcal{C}$.

2. Each edge in $\Gamma$ is contained in exactly 10 of the cliques in $\mathcal{C}$.

3. Each $k$-clique in $\Gamma$ with $3 \leq k \leq 5$ is contained in exactly one of the cliques in $\mathcal{C}$.

4. There are no $k$-cliques in $\Gamma$ of size $k>5$.

Proof. The group $G$ acts transitively on both the vertices and the edges of $\Gamma$. Consequently, each of the $275 \cdot 112 / 2=15400$ edges of $\Gamma$ occur in $15400 \cdot 10 / 15400=10$ of the cliques in $\mathcal{C}$, and each of the 275 vertices of $\Gamma$ occur in $15400 \cdot 5 / 275=280$ of the cliques in $\mathcal{C}$.

Since $\Gamma$ is a pseudogeometric $(4,27,2)$-graph, each vertex not in a given 5 -clique is adjacent to exactly two vertices in that clique. It is shown in [15] that each 3-clique is contained in some 5-clique (or see the Appendix). This 5-clique $C$ is unique, since otherwise there would be a vertex outside $C$ that is adjacent to at least three vertices of $C$, which is not possible. This implies that also each 4-clique is contained in a unique 5 -clique and that there can be no clique in $\Gamma$ of size greater than 5 .

The existence problem for a $\operatorname{pg}(4,27,2)$ boils down to the problem of finding a subset of size 1540 of the set $\mathcal{C}$ of 5 -cliques such that each edge of the McLaughlin graph is in exactly one of the chosen 5-cliques. For this, the framework of exact cover [12, 13] could be considered, and indeed this approach is used in [17]. However, many further ideas are needed to solve the existence problem of a McLaughlin geometry, even with the large available computing resources. Indeed, it is clear from earlier studies [15, 17, 25] that constructing a McLaughlin geometry, or proving nonexistence, is a big challenge. As with other similar challenging problems [11, care has to be taken to find an efficient approach that utilizes the available symmetries.

\section{The general approach}

Our general strategy follows that described by Reichard [22]. This strategy was independently devised by the second author, and implemented in his GRAPE package [26] function PartialLinearSpaces, which can classify partial linear spaces, including partial geometries, with given parameters and point graph, up to isomorphism. In the present work, however, we needed 
to employ new ideas and enhancements, implemented on a powerful 256core computer cluster, to be able finally to solve the existence problem of a McLaughlin geometry.

We first define the concept of a bundle. If $p$ is a point in a McLaughlin geometry, then the 28 lines through $p$ meet pairwise only in $p$, for otherwise, there would be two distinct points on more than one line. Where $p$ is a vertex in the McLaughlin graph $\Gamma$ (we also call $p$ a point), then a bundle through $p$ is a set of 285 -cliques whose pairwise intersection is $\{p\}$.

Our basic approach for an exhaustive search for a McLaughlin geometry is to consider the points $1,2, \ldots, 275$ of $\Gamma$, and given chosen bundles through $i-1$ chosen points, $p_{1}, \ldots, p_{i-1}$, to choose a new point $p_{i}$ (which may depend on the points and bundles chosen so far), then consider all the possible bundles through $p_{i}$. Some bundles through $p_{i}$ may be incompatible with previous choices, or may not be necessary to consider by symmetry or since they were eliminated from consideration in a previous case, but for each bundle through $p_{i}$ that it is necessary to consider, we continue the search using a new point $p_{i+1}$ not in $\left\{p_{1}, \ldots, p_{i}\right\}$. The full search starts with $i=1$.

\subsection{Classifying bundles}

Since the group $G$ acts transitively on the points, the first point $p_{1}$ can be chosen arbitrarily, so we choose $p_{1}=1$, and start by classifying the bundles through this point, up to the action of the stabilizer $U$ in $G$ of $p_{1}$. This stabilizer has shape $U_{4}(3) .2$ [19] and order $|G| / 275=2^{8} \cdot 3^{6} \cdot 5 \cdot 7=6531840$.

There are exactly 17729280 bundles through $p_{1}$, forming just $36 U$-orbits. This was already discovered by Pech and Reichard [21, Sect. 8.2], but no further details about the structures were given in that study.

We determined the $36 U$-orbits of the 17729280 distinct bundles through $p_{1}$ using exact cover [12]: each 5-clique that contains $p_{1}$ covers the four other points on the candidate line, and we need to determine the sets (of size 28) of such 5-cliques that cover each the 112 vertices adjacent to $p_{1}$ exactly once. The Orbit-Stabilizer theorem was applied for validating the results; cf. [11, Sect. 10.2]. More precisely, it was checked that the number of bundles in each orbit determined during the search is $6531840 /|A|$, where $|A|$ is the order of the stabilizer in $U$ of an orbit representative.

We remark that the bundles through $p_{1}$ correspond to the "spreads" of the $\operatorname{pg}(3,9,1)$ (or generalised quadrangle $\mathrm{GQ}(3,9)$ ), whose point graph is the induced subgraph $\Delta$ on the neighbors in $\Gamma$ of $p_{1}$, and that these spreads form just 26 orbits under the action of the automorphism group of $\Delta$, of shape $U_{4}(3) . D_{8}$ (see [2] and [21, Sect. 8.2]). 
We use the 36 lex-min $U$-orbit representatives of the bundles in subsequent computations. This is an important detail, since, for example, references to specific points are not valid for arbitrary representatives. The natural order $1,2, \ldots, 275$ of the points naturally induces a lexicographic order on the candidate lines (which are sets of points), and so also on the sets of candidate lines. The lex-min $U$-orbit representatives of the bundles through $p_{1}$ were computed independently by both authors. The second author used GRAPE [26], making particular use of its included function SmallestImageSet [16, written by Steve Linton, which, given a permutation group $A \leq S_{n}$ and a subset $S$ of $\{1, \ldots, n\}$, determines the lex-min set in the $A$-orbit of $S$ (without explicitly constructing this orbit).

To find the automorphism groups of graphs, we use nauty [18] in the current work. Isomorphism and automorphism problems for incidence structures encountered (such as sets of lines) are also conveniently handled by nauty after standard reductions. Furthermore, the SmallestImageSet function in GRAPE is very useful for testing isomorphism when isomorphism corresponds to two sets being in the same group orbit.

Note that, due to the transitivity of $G$ on the points, our 36 lex-min $U$ orbit representatives form a set of representatives for the $G$-orbits of all the bundles through all the points. We consider two bundles to be isomorphic if they are in the same $G$-orbit. In the subsequent computations, it will be important to be able to distinguish between the 36 isomorphism classes of bundles with some invariant that can be computed reasonably quickly. Brouwer [2] describes the following invariant, which partitions the bundles into 11 classes.

Count the number of 4 -sets $F$ of lines in the bundle through $p$ that satisfy the following property: there are four additional candidate lines through $p$ that intersect each line in $F$ in some point other than $p$. It follows that these four additional candidate lines must pairwise intersect only in $p$, for otherwise, we would have a triangle in the generalised quadrangle whose point graph is induced on the neighbors in $\Gamma$ of $p$. Hence, with $p$ removed, the eight lines consisting of a 4 -subset $F$ of the bundle together with four candidate lines meeting each element of $F$ in a point form a $4 \times 4$ "grid". The value of this invariant is between 0 and 63 for the 36 isomorphism classes. (This subdivision was used also by Reichard.)

We shall now consider a new invariant that is able to distinguish between all 36 isomorphism classes of bundles. Let $p$ be a point and $P$ be the set of points that are not adjacent in $\Gamma$ to $p$. Given a bundle through $p$ consider the set of candidate lines $L$ that do not contain $p$ but intersect two points of some line in the bundle through $p$. The number of such candidate lines is obviously $28 \cdot 6 \cdot 9=1512$. Each candidate line in $L$ covers three pairs 
of points in $P$. Moreover, each pair of points in $P$ is covered by 0 to 10 candidate lines in $L$.

The values $t_{1}, \ldots, t_{10}$, where $t_{i}$ is the number of pairs of points in $P$ covered exactly $i$ times are now invariants. Clearly $\sum_{i=1}^{10} i t_{i}=1512 \cdot 3=4536$. The tuple $\left(t_{1}, t_{2}, \ldots, t_{10}\right)$ turns out to be an almost complete invariant actually, it will suffice to use only a part of the tuple, for example, $\left(t_{1}, t_{2}\right)$ leaving only two pairs of isomorphism classes open.

To make the invariant complete one may further have a look at the candidate lines $L^{\prime}$ that intersect exactly two of the lines in the bundle in one point each. Let $L^{\prime \prime} \subseteq L^{\prime}$ be the subset of lines that do not intersect any of the sets in $\{C \cap P: C \in L\}$ in more than one point. Finally, find a line in the bundle that intersects the fewest number $s$ of lines in $L^{\prime \prime}$, and use $s$ in the invariant.

The following invariants of the 36 isomorphism classes are listed in Table 1 the old invariant (Old), the values $s, t_{1}, \ldots, t_{10}$ for the new invariant, and the order $\mid$ Stab $\mid$ of the stabilizer in $G$ of a representative bundle.

Table 1 shows that six of the 36 representative bundles have $t_{10}>0$. Hence, for each of these six bundles, there is a pair of points which does not occur in any feasible candidate line (as this pair is only covered by candidate lines which cannot be lines in a McLaughlin geometry containing that bundle). The fact that these six cases cannot lead to a McLaughlin geometry was discovered already by Reichard (as noted in [25, p. 252]), but the specific details were never published [23].

We remark that, given a bundle, a $113 \times 1540$ part of the $275 \times 1540$ pointline incidence matrix of a McLaughlin geometry $\mathcal{G}$ is determined. We can see this making use of the fact that every point in $\Gamma$ not on a given candidate line $C \in \mathcal{C}$ must be adjacent to exactly two points in $C$. This implies that if $C_{1}$ and $C_{2}$ are distinct candidate lines in our bundle with common point $p$, and $q \neq p$ is a point in $C_{1}$, then $q$ is adjacent to just one point other than $p$ in $C_{2}$, and this yields a total of $1512=\left(\begin{array}{c}28 \\ 2\end{array}\right) 4$ edges to be covered once each by the lines of $\mathcal{G}$ not in our bundle. Consequently, the points of the bundle induce a partial linear space having 28 lines of size 5 and 1512 lines of size 2 .

\subsection{Classifying pairs of bundles}

Now, for each of the 30 lex-min representative bundles $B$ through $p_{1}=1$ that have $t_{10}=0$, we will choose a new point $p_{2}$ and determine all bundles through $p_{2}$ that are compatible with $B$, up to the action of the stabilizer of $p_{2}$ in the $G$-stabilizer of $B$. Two bundles are said to be compatible if no line of the first intersects any line of the second in exactly two points.

The order in which the points are completed, and in particular the choice 
Table 1: Isomorphism classes of bundles

\begin{tabular}{|c|c|c|c|c|c|c|c|c|c|c|c|c|c|}
\hline $\mathrm{Nr}$ & Old & $s$ & $t_{1}$ & $t_{2}$ & $t_{3}$ & $t_{4}$ & $t_{5}$ & $t_{6}$ & $t_{7}$ & $t_{8}$ & $t_{9}$ & $t_{10}$ & $\mid$ Stab| \\
\hline 1 & 0 & 64 & 1730 & 762 & 288 & 97 & 6 & 0 & 0 & 0 & 0 & 0 & 6 \\
\hline 2 & 0 & 80 & 1603 & 840 & 336 & 35 & 21 & 0 & 0 & 0 & 0 & 0 & 7 \\
\hline 3 & 0 & 80 & 1568 & 910 & 266 & 70 & 14 & 0 & 0 & 0 & 0 & 0 & 14 \\
\hline 4 & 0 & 84 & 1568 & 910 & 266 & 70 & 14 & 0 & 0 & 0 & 0 & 0 & 14 \\
\hline 5 & 0 & 68 & 1673 & 875 & 231 & 70 & 28 & 0 & 0 & 0 & 0 & 0 & 14 \\
\hline 6 & 0 & 76 & 1736 & 770 & 301 & 70 & 7 & 7 & 0 & 0 & 0 & 0 & 14 \\
\hline 7 & 0 & 70 & 1686 & 981 & 117 & 93 & 18 & 9 & 3 & 0 & 0 & 0 & 18 \\
\hline 8 & 0 & 78 & 1626 & 837 & 360 & 15 & 0 & 9 & 6 & 0 & 0 & 0 & 18 \\
\hline 9 & 0 & 54 & 1800 & 810 & 207 & 108 & 0 & 0 & 9 & 0 & 0 & 0 & 18 \\
\hline 10 & 0 & 64 & 1640 & 873 & 270 & 60 & 18 & 0 & 0 & 0 & 0 & 1 & 18 \\
\hline 11 & 0 & 82 & 1589 & 903 & 231 & 112 & 0 & 0 & 0 & 0 & 0 & 0 & 21 \\
\hline 12 & 0 & 84 & 1589 & 903 & 231 & 112 & 0 & 0 & 0 & 0 & 0 & 0 & 21 \\
\hline 13 & 1 & 66 & 1728 & 852 & 208 & 102 & 0 & 12 & 0 & 0 & 0 & 0 & 16 \\
\hline 14 & 1 & 50 & 1624 & 994 & 184 & 32 & 32 & 14 & 0 & 0 & 0 & 0 & 16 \\
\hline 15 & 1 & 73 & 1792 & 752 & 272 & 92 & 0 & 8 & 0 & 1 & 0 & 0 & 16 \\
\hline 16 & 1 & 52 & 1824 & 739 & 264 & 92 & 8 & 4 & 0 & 0 & 0 & 1 & 16 \\
\hline 17 & 1 & 73 & 1824 & 840 & 216 & 30 & 24 & 24 & 0 & 0 & 0 & 0 & 48 \\
\hline 18 & 1 & 27 & 1896 & 780 & 168 & 120 & 0 & 12 & 0 & 3 & 0 & 0 & 48 \\
\hline 19 & 2 & 70 & 1708 & 832 & 264 & 57 & 26 & 0 & 2 & 0 & 0 & 0 & 2 \\
\hline 20 & 3 & 73 & 1648 & 855 & 285 & 51 & 21 & 0 & 2 & 0 & 0 & 0 & 3 \\
\hline 21 & 3 & 59 & 1700 & 870 & 240 & 64 & 12 & 6 & 0 & 3 & 0 & 0 & 24 \\
\hline 22 & 3 & 50 & 1764 & 831 & 240 & 62 & 12 & 12 & 0 & 0 & 0 & 1 & 24 \\
\hline 23 & 4 & 60 & 1773 & 726 & 315 & 72 & 12 & 3 & 0 & 0 & 0 & 0 & 6 \\
\hline 24 & 6 & 60 & 1717 & 789 & 309 & 47 & 18 & 6 & 0 & 0 & 0 & 0 & 6 \\
\hline 25 & 7 & 82 & 1520 & 1008 & 232 & 52 & 8 & 4 & 0 & 4 & 0 & 0 & 16 \\
\hline 26 & 7 & 68 & 1664 & 876 & 272 & 40 & 16 & 8 & 0 & 2 & 0 & 0 & 32 \\
\hline 27 & 7 & 60 & 1664 & 994 & 144 & 80 & 16 & 0 & 0 & 4 & 0 & 2 & 32 \\
\hline 28 & 7 & 89 & 1368 & 1080 & 264 & 36 & 0 & 12 & 0 & 0 & 0 & 0 & 48 \\
\hline 29 & 9 & 0 & 1584 & 1242 & 0 & 36 & 0 & 54 & 0 & 0 & 0 & 0 & 432 \\
\hline 30 & 9 & 38 & 1968 & 837 & 0 & 216 & 0 & 0 & 0 & 0 & 0 & 3 & 432 \\
\hline 31 & 15 & 64 & 1728 & 808 & 280 & 60 & 8 & 12 & 0 & 0 & 0 & 0 & 16 \\
\hline 32 & 15 & 48 & 1704 & 792 & 312 & 60 & 0 & 12 & 0 & 0 & 0 & 0 & 48 \\
\hline 33 & 15 & 78 & 1664 & 908 & 224 & 76 & 0 & 8 & 0 & 4 & 0 & 0 & 64 \\
\hline 34 & 15 & 72 & 1792 & 948 & 96 & 112 & 0 & 12 & 0 & 0 & 0 & 4 & 192 \\
\hline 35 & 31 & 64 & 1824 & 792 & 192 & 120 & 0 & 12 & 0 & 0 & 0 & 0 & 192 \\
\hline 36 & 63 & 0 & 2016 & 756 & 0 & 252 & 0 & 0 & 0 & 0 & 0 & 0 & 12096 \\
\hline
\end{tabular}


of $p_{2}$ for each chosen bundle through $p_{1}$, are crucial. We shall now have a closer look at the cases when $p_{1}$ and $p_{2}$ are adjacent and nonadjacent.

When $p_{1}$ and $p_{2}$ are adjacent, their completion has a line $\ell=\left\{p_{1}, p_{2}, a, b, c\right\}$. Given the bundle through $p_{1}$, out of the 280 candidate lines through $p_{2}$, the infeasible lines through $p_{2}$ are exactly those lines that contain $p_{1}$ and one other point of $\ell$. This gives a total of $280-(1+4 \cdot 9)=243$ feasible lines.

One may also see that the bundle through $p_{1}$ reduces the number of possible bundles through $p_{2}$ from 17729280 to 1772928 , that is, by a factor of exactly 10. This follows from the fact that given $p_{1}$, out of the ten possibilities for the line through $p_{1}$ and $p_{2}$, one has been fixed. Since the $G$-stabilizer of $p_{2}$ is transitive on the set of 5-cliques containing that point, and the bundle through $p_{1}$ does not restrict any candidate lines through $p_{2}$ that intersect $\ell$ only in that point, the reduction factor is indeed 10 .

If $p_{1}$ and $p_{2}$ are nonadjacent, then the vertices in $\Gamma$ adjacent to both points induce the Gewirtz graph, which is the unique $(56,10,0,2)$-srg (see [3]). Every candidate line through $p_{1}$ and $p_{2}$ intersects the vertex set of that graph in exactly 2 vertices, and a bundle partitions it into 28 pairs. Consequently, given a bundle through $p_{1}$, there are $280-28=252$ feasible lines for a bundle through $p_{2}$. (Recall that a 3 -clique is in a unique candidate line by Lemma 1.)

Although the number of feasible lines through $p_{2}$ is smaller when $p_{1}$ and $p_{2}$ are adjacent than when they are nonadjacent, it turns out that the number of compatible bundles is smaller in practice when they are nonadjacent. We do not have a formal argument for this, but the following probabilistic approach gives a result surprisingly close to experimental results.

The bundles through $p_{1}$ and $p_{2}$ each cover 28 edges out of the 280 edges in the Gewirtz graph. The probability that two sets of 28 randomly chosen edges are distinct is

$$
\frac{\left(\begin{array}{c}
252 \\
28
\end{array}\right)}{\left(\begin{array}{c}
280 \\
28
\end{array}\right)} \approx 0.04454 .
$$

This value is smaller than $1 / 10=0.1$. Although this reasoning cannot be directly applied to the case of more than two completed points, practical experiments show that independent sets are indeed a proper choice when extending larger set of points.

When choosing the second point $p_{2}$ to complete amongst those vertices (other than $p_{1}$ ) that are not adjacent to $p_{1}$, the possible choices correspond to the orbits of these points under the stabilizer in $G$ of the given bundle $B$ through $p_{1}$. Isomorph rejection will be carried out on the compatible bundles through the chosen $p_{2}$, using the action of the stabilizer $K$ of $p_{2}$ in 
the $G$-stabilizer of $B$.

Let $\mid$ Stab $\mid$ be the order of the stabilizer in $G$ of the bundle $B$ through $p_{1}=1$, and let $l$ be the length of that group's point-orbit containing $p_{2}$. Then the group $K$ above will have order $\mid$ Stab $\mid / l$. Let $N$ be the number of bundles through $p_{2}$ that are compatible with the first bundle $B$. It turns out that most sets of two bundles have only trivial symmetries, so the number of solutions up to symmetry is approximately $N_{1}:=N l / \mid$ Stab $\mid$. The point $p_{2}$ is chosen so that $N_{1}$ is minimized. The exact number of solutions up to symmetry is denoted by $N_{2}$. The Orbit-Stabilizer theorem is used also here to validate the results. For each of the 30 lex-min isomorphism class representative bundles through $p_{1}$ which were not rejected in the first step, the compatible bundles through the chosen $p_{2}$ were classified and their lexmin $K$-orbit representatives were computed by both authors independently, using different software and computers, the second author using GRAPE and SmallestImageSet on a Linux desktop PC. The results were compared and found to be exactly the same.

In Table 2 we now list information about each case and chosen point $p_{2}$, ignoring the six cases that were rejected in the first step. The notation given above is used for the columns of the table. The last two columns, $N_{3}$ and $H$, will be discussed in the next subsection.

\subsection{Completing the search}

We have already seen that six types of bundles need not be considered at all in the computer search, splitting the overall search into 30 cases, with one case for each of the remaining bundle types, where we shall be searching for a McLaughlin geometry containing a bundle of that type. It is clear that after a case for a given bundle type is completed, that bundle type may be cancelled, that is, eliminated from consideration in the cases of the search to follow. A bundle of a cancelled type can be recognised via our bundle isomorphism class invariant. The order in which the cases are considered will have an impact on the overall running time.

The value of $N_{2}$ in Table 2 gives a rough estimate of the time it takes to complete the cases (ignoring possible cancelled cases). It further turns out that the impact of a cancelled bundle type on the subsequent cases is roughly inversely proportional to $\mid$ Stab $\mid$. Consequently, a reasonable heuristic for ordering the parts is via the value $H:=N_{2} \mid$ Stab $\mid$; this value is given in the last column of Table 2. (Earlier approaches of using invariants and splitting up the search in many parts are, for example, [9, 10].)

We define an order for the parts based on $H$, but put case 36 early since that is a very small case and good for testing. The order is as follows 
Table 2: Choices for second point

\begin{tabular}{rrrrrrrrr}
\hline Nr & $\mid$ Stab & $p_{2}$ & $N$ & $l$ & $N_{1}$ & $N_{2}$ & $N_{3}$ & $H$ \\
\hline 1 & 6 & 68 & 940320 & 1 & 156720 & 157760 & 72811 & 946560 \\
2 & 7 & 161 & 750620 & 1 & 107231 & 107246 & 74493 & 750722 \\
3 & 14 & 16 & 1072194 & 1 & 76585 & 77641 & 25505 & 1086974 \\
4 & 14 & 79 & 1055464 & 1 & 75390 & 76474 & 26850 & 1070636 \\
5 & 14 & 87 & 1041676 & 1 & 74405 & 75034 & 27941 & 1050476 \\
6 & 14 & 159 & 1078178 & 1 & 77013 & 78153 & 23269 & 1094142 \\
7 & 18 & 136 & 1159506 & 1 & 64417 & 65666 & 17619 & 1181988 \\
8 & 18 & 69 & 1032450 & 3 & 172075 & 172266 & 10186 & 3100788 \\
9 & 18 & 56 & 1089000 & 3 & 181500 & 181742 & 7044 & 3271356 \\
10 & & & & & & & & \\
11 & 21 & 27 & 1356738 & 1 & 64607 & 64610 & 15233 & 1356810 \\
12 & 21 & 79 & 1356738 & 1 & 64607 & 64610 & 14147 & 1356810 \\
13 & 16 & 106 & 431840 & 1 & 26990 & 27412 & 25365 & 438592 \\
14 & 16 & 172 & 583384 & 1 & 36461 & 37204 & 33452 & 595264 \\
15 & 16 & 118 & 504528 & 2 & 63066 & 63066 & 24992 & 1009056 \\
16 & & & & & & & & \\
17 & 48 & 18 & 851600 & 6 & 106450 & 106450 & 846 & 5109600 \\
18 & 48 & 126 & 565584 & 3 & 35349 & 35578 & 6749 & 1707744 \\
19 & 2 & 220 & 894832 & 1 & 447416 & 448417 & 288705 & 896834 \\
20 & 3 & 180 & 600438 & 1 & 200146 & 200146 & 176116 & 600438 \\
21 & 24 & 190 & 1209360 & 1 & 50390 & 50718 & 12620 & 1217232 \\
22 & & & & & & & & \\
23 & 6 & 68 & 776563 & 3 & 388282 & 388842 & 52122 & 2333052 \\
24 & 6 & 240 & 714792 & 1 & 119132 & 120096 & 90940 & 720576 \\
25 & 16 & 16 & 1053336 & 2 & 131667 & 132016 & 20285 & 2112256 \\
26 & 32 & 3 & 402504 & 4 & 50313 & 50313 & 10310 & 1610016 \\
27 & & & & & & & & \\
28 & 48 & 37 & 571676 & 6 & 71459 & 71683 & 1348 & 3440784 \\
29 & 432 & 8 & 1048560 & 36 & 87380 & 87778 & 67 & 37920096 \\
30 & & & & & & & & \\
31 & 16 & 42 & 457198 & 4 & 114300 & 114670 & 20561 & 1834720 \\
32 & 48 & 16 & 485100 & 6 & 60637 & 60746 & 4104 & 2915808 \\
33 & 64 & 73 & 1216976 & 2 & 38030 & 38207 & 2705 & 2445248 \\
34 & & & & & & & & \\
35 & 192 & 38 & 402712 & 12 & 25169 & 25441 & 250 & 4884672 \\
36 & 12096 & 8 & 396552 & 36 & 1180 & 1231 & 1160 & 14890176 \\
\hline & & & & & & & &
\end{tabular}


(including the six cases that need not be considered at all at this stage):

$$
\begin{aligned}
& 10 \rightarrow 16 \rightarrow 22 \rightarrow 27 \rightarrow 30 \rightarrow 34 \rightarrow 36 \rightarrow 13 \rightarrow 14 \rightarrow 20 \rightarrow 24 \rightarrow 2 \rightarrow \\
& 19 \rightarrow 1 \rightarrow 15 \rightarrow 5 \rightarrow 4 \rightarrow 3 \rightarrow 6 \rightarrow 7 \rightarrow 21 \rightarrow 11 \rightarrow 12 \rightarrow 26 \rightarrow \\
& 18 \rightarrow 31 \rightarrow 25 \rightarrow 23 \rightarrow 33 \rightarrow 32 \rightarrow 8 \rightarrow 9 \rightarrow 28 \rightarrow 35 \rightarrow 17 \rightarrow 29
\end{aligned}
$$

Having decided the order, the structures enumerated under $N_{2}$ in Table 2 can immediately be reduced by removing the bundles whose types will be cancelled before a given case is handled. The numbers of remaining structures, which are seeds for the final stage, are given in column $N_{3}$ of the same table.

The idea of considering independent sets of points to complete turned out to have further benefits when programming the search. For various reasons related to speed and memory requirement, we want to predetermine a set of points to consider in the search. Here we obviously rely on the assumption that it suffices to consider a relatively small set of points to prove nonexistence. Results by Reichard [22, Sect. 5.3] give an indication that this is indeed the case.

There are many independent sets in $\Gamma$, so the choice of points is not obvious. We use the following approach. We first find the set $S$ of points other than $p_{1}$ and $p_{2}$ that are neither adjacent to $p_{1}$ nor to $p_{2}$. From the parameters of $\Gamma$, we know that $|S|=105$. Let $B(p)$ be the number of bundles through $p$ that are compatible with both of the two bundles through $p_{1}$ and $p_{2}$. For a given $n$, we now find an independent set $I \subseteq S,|I|=n$ that minimizes $\sum_{p \in I} B(p)$. We use $n=9$. (Since all pairs of nonadjacent vertices are in one orbit of the automorphism group of $\Gamma$, the number of independent sets $I$ of a given size to consider will not depend on the partial solution; for $n=9$, it is 18579960.) To get an upper bound on the size of the data structures needed, one may further require that $\max _{p \in I} B(p)<u$, for some constant $u$, when minimizing $\sum_{p \in I} B(p)$. We here use this additional condition with $u=10^{5}$.

The core of the search is basic backtrack search. Throughout the search, the candidate bundles through each of the considered points are maintained. On each level of the search, the point to consider is first determined. Specifically, a point with the fewest candidates is chosen. All candidate bundles for that point are then considered in order. For each fixed bundle, the candidate sets for the other points are reduced and the procedure described here is recursively repeated.

The core task of the backtrack search is to determine whether two bundles are compatible. At one extreme, one could preprocess every pair of candidate bundles and tabulate one bit for the result (this is essentially about determining the edges of a graph in which a clique search is to be carried out). 
However, with, say, 300000 candidates in total, the task of determining a matrix with $\left(3 \cdot 10^{5}\right)^{2}=9 \cdot 10^{10}$ entries is time-consuming and, in particular, a substantial amount of memory is required.

At the other extreme, one may process each pair of bundles as they appear in the search. This requires that up to $28 \cdot 28=784$ pairs of lines be compared, which is a rather large constant. Actually, this approach was taken when carrying out a partial check of the results with an independent program. Between these extremes, there are several other possibilities, out of which the following was chosen.

Assume that we compare a bundle through point $p$ with a bundle through a nonadjacent point $p^{\prime}$. Now consider the set $P$ of points that will lie on lines through $p$ as well as lines through $p^{\prime}$. The size of $P$ equals the parameter $\mu=56$ for $\Gamma$. We have seen earlier that $P$ induces a $(56,10,0,2)-\operatorname{srg}$ in $\Gamma$, the Gewirtz graph, which has $56 \cdot 10 / 2=280$ edges. The 28 lines through $p$ and $p^{\prime}$ will each contain exactly 28 of the 280 edges. We now have the information needed for the data structure.

Let $I$ be the (independent) set of points to be completed in the search. For each pair $(B, p)$, where $B$ is a candidate bundle through $p^{\prime}$, such that $p^{\prime}, p \in I$ and $p^{\prime} \neq p$, determine and save a 280 -bit vector with 28 1s indicating which pairs of points of lines in $B$ are in the common neighborhood of $p^{\prime}$ and $p$. When comparing two bundles, it now suffices to compare two 280-bit vectors. This can be implemented by checking whether five AND operations on 64-bit integers all produce 0 . This very fast method of determining compatibility of bundles was crucial to the success of our search.

\section{Results}

The backtrack search described in Section 3.3 was carried out on a 256-core cluster with 2.4-GHz Intel Xeon E5-2665 processors. As the $n=9$ points could not be completed in any of the cases, we have the following result.

Theorem 1. There is no partial geometry with parameters $(s, t, \alpha)=(4,27,2)$, that is, there is no McLaughlin geometry.

For the 30 cases, we collect the main data in Table 3. We give the total run time (in core-days), run time per instance of the case (the number of instances is $N_{3}$ in Table 2), and the maximum number of points completed in the search. The total hardware run-time was approximately 250 coreyears, but since in some parts of the search virtual cores were used, the times per case given in Table 3 sum to rather more than this. 
The length of our computer search was at the borderline of what was doable with the resources available to the authors. Consequently, a lot of effort was put into both the general approach and the specific details described in this paper. In retrospect, the authors feel that more effort should still have been put on one part, namely on deciding the order in which the bundle types are handled. The order used, displayed in the beginning of Section 3.3, was obtained with a heuristic, but additional computational experiments might have led to a different order and faster computations.

Correctness is one of the major issues of computer searches [14, 24]. There are effective validation methods whenever the solution set for an instance of a classification problem is nonempty [11, Sect. 10.2], which can be applied to the initial phases of this search as we have seen. However, for the final phase, which produces no solutions, the situtation is different. Also notice that the idea of considering subcases to avoid redundant computations, which was crucial for the success of the work, means that the following argument cannot be used: if something exists, then it can been found in many ways.

Two measures were taken to minimize the probability of error. A computing cluster with error-correcting code (ECC) memory was used to minimize the probability of hardware error. Moreover, the results of a different, basic (and therefore slow) implementation of the final backtrack search were compared with the main implementation for a part of the search tree (comparing very specific details).

Having answered the existence question of a McLaughlin geometry in the negative, one may continue and ask for the largest number of 5-cliques (candidate lines) in the McLaughlin graph such that no two distinct such cliques intersect in two points. Mathon [17] found such packings of size 1120, on which one could try to improve. Finding the exact answer for this problem does not seem to be within reach.

\section{Appendix}

In this Appendix we present a GAP/GRAPE logfile specifying the construction of our standard fixed copy of the McLaughlin graph $\Gamma$, used in all our computations. The group $G$, obtained from the GAP library of primitive groups and given here by explicit generators, is our standard copy of the automorphism group of $\Gamma$. The vertex-set of $\Gamma$ is $\{1, \ldots, 275\}$, and the edge-set is the $G$-orbit of $\{1,2\}$.

Checks are included showing $G$ to have the right order, $\Gamma$ to be a strongly regular graph with the correct parameters, $G=\operatorname{Aut}(\Gamma)$, and that the maximal cliques of $\Gamma$ (maximal with respect to containment) are precisely the 
Table 3: Search data

\begin{tabular}{|c|c|c|c|}
\hline $\mathrm{Nr}$ & Time (d) & Time/Instance (min) & Max. level \\
\hline 1 & 2325 & 46.0 & 6 \\
\hline 2 & 13958 & 269.8 & 7 \\
\hline 3 & 217 & 12.3 & 6 \\
\hline 4 & 264 & 14.2 & 6 \\
\hline 5 & 301 & 15.5 & 6 \\
\hline 6 & 162 & 10.0 & 6 \\
\hline 7 & 100 & 8.2 & 6 \\
\hline 8 & 45 & 6.4 & 5 \\
\hline 9 & 30 & 6.2 & 4 \\
\hline \multicolumn{4}{|l|}{10} \\
\hline 11 & 88 & 8.3 & 6 \\
\hline 12 & 76 & 7.8 & 5 \\
\hline 13 & 6942 & 394.1 & 7 \\
\hline 14 & 6619 & 284.9 & 7 \\
\hline 15 & 302 & 17.4 & 6 \\
\hline \multicolumn{4}{|l|}{16} \\
\hline 17 & 4 & 6.4 & 3 \\
\hline 18 & 34 & 7.3 & 5 \\
\hline 19 & 42822 & 213.6 & 7 \\
\hline 20 & 78792 & 644.2 & 8 \\
\hline 21 & 67 & 7.7 & 6 \\
\hline \multicolumn{4}{|l|}{22} \\
\hline 23 & 271 & 7.5 & 5 \\
\hline 24 & 15988 & 253.2 & 7 \\
\hline 25 & 86 & 6.1 & 5 \\
\hline 26 & 50 & 7.0 & 6 \\
\hline \multicolumn{4}{|l|}{27} \\
\hline 28 & 6 & 6.3 & 4 \\
\hline 29 & 1 & 5.9 & 2 \\
\hline \multicolumn{4}{|l|}{30} \\
\hline 31 & 96 & 6.7 & 5 \\
\hline 32 & 18 & 6.2 & 5 \\
\hline 33 & 10 & 5.5 & 5 \\
\hline \multicolumn{4}{|l|}{34} \\
\hline 35 & 1 & 5.8 & 3 \\
\hline 36 & 66 & 82.3 & 6 \\
\hline
\end{tabular}


cliques of size 5 , of which there is a single $G$-orbit, which has size 15400 .

gap> LoadPackage ("grape") ;

Loading GRAPE 4.6.1 (GRaph Algorithms using PErmutation groups)

by Leonard H. Soicher (http://www.maths.qmul.ac.uk/ leonard/).

Homepage: http://www.maths.qmul.ac.uk/ leonard/grape/

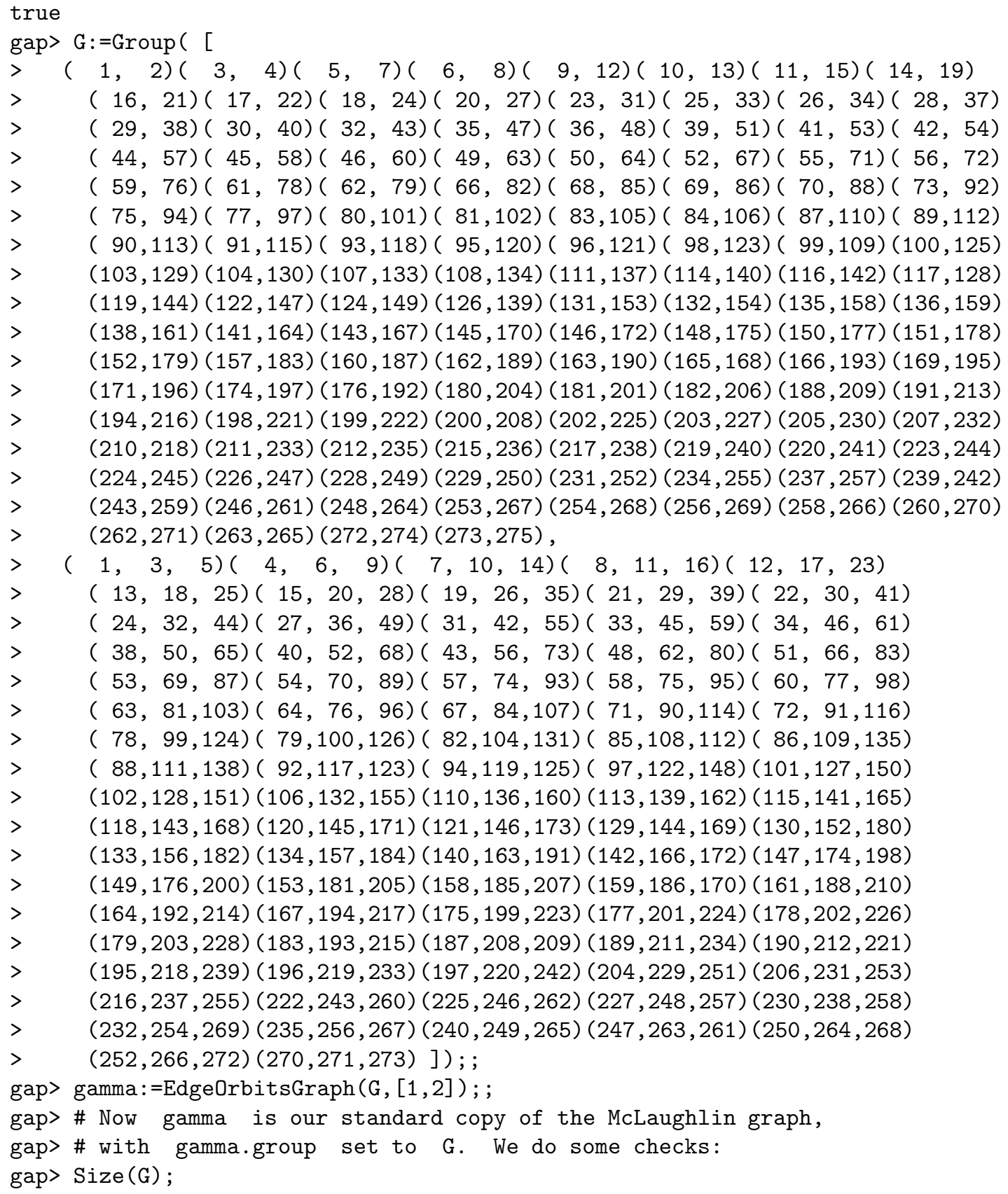




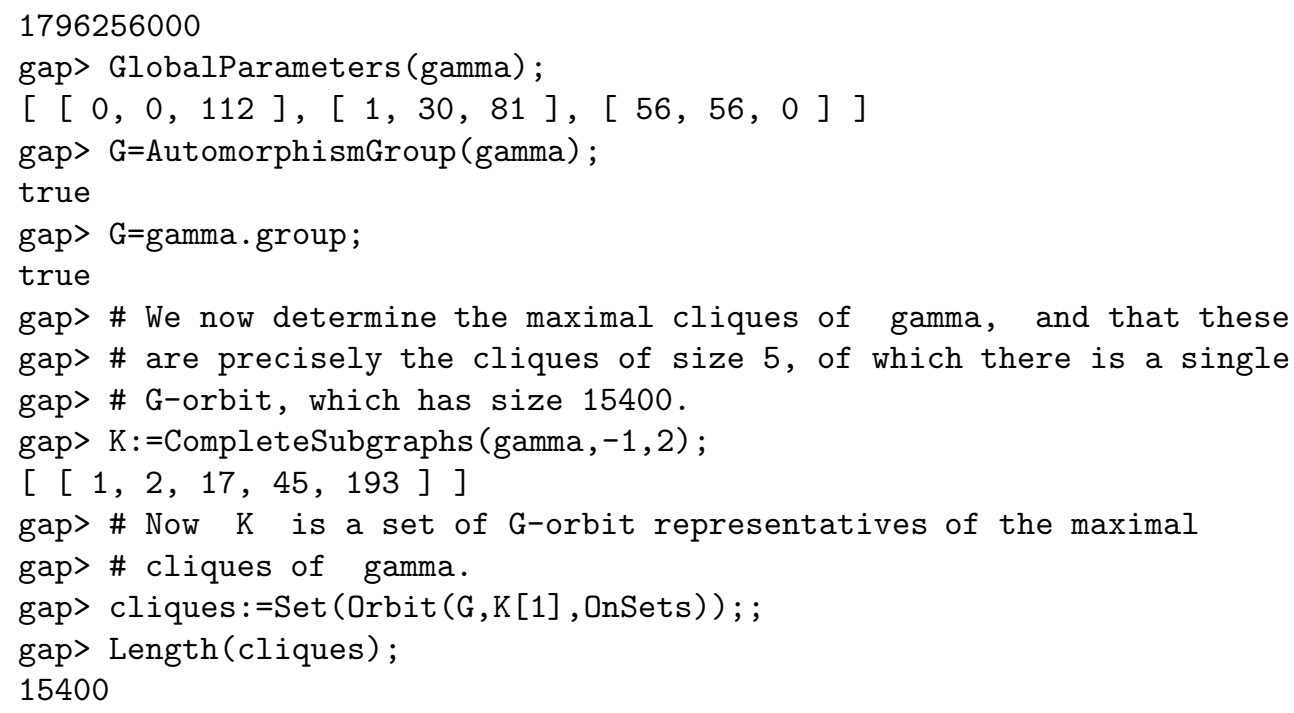

\section{Acknowledgements}

The first author was supported in part by the Academy of Finland, Project No. 289002. We thank Frank De Clerck for making the second author aware, about 25 years ago, of the interesting and challenging problem of the existence of a McLaughlin geometry. Andries Brouwer's web pages with extensive data on many interesting graphs, especially strongly regular graphs, were invaluable in the development of the algorithms and heuristics.

\section{References}

[1] R. C. Bose, Strongly regular graphs, partial geometries and partially balanced designs, Pacific J. Math. 13 (1963), 389-419.

[2] A. E. Brouwer, The 1st subconstituent of the McLaughlin graph $\left(O^{-}(6,3)\right)$ on 112 vertices, http://www.win.tue.nl/ aeb/graphs/U4_3.html

[3] A. E. Brouwer and W. H. Haemers, The Gewirtz graph: an exercise in the theory of graph spectra, European J. Combin. 14 (1993), 397-407.

[4] P. J. Cameron, J.-M. Goethals, and J. J. Seidel, Strongly regular graphs having strongly regular subconstituents, J. Algebra 55 (1978), 257-280. 
[5] F. De Clerck and H. Van Maldeghem, Some classes of rank 2 geometries, in: F. Buekenhout (Ed.), Handbook of Incidence Geometry. Buildings and Foundations, North-Holland, Amsterdam, 1995, pp. 433-475.

[6] The GAP Group, GAP - Groups, Algorithms, and Programming, Version 4.7.6, 2014, http://www.gap-system.org/

[7] J.-M. Goethals and J. J. Seidel, The regular two-graph on 276 vertices, Discrete Math. 12 (1975), 143-158.

[8] W. Haemers, A new partial geometry constructed from the HoffmanSingleton graph, in: P. J. Cameron, J. W. P. Hirschfeld, and D. R. Hughes (Eds.), Finite Geometries and Designs. Proceedings of the Second Isle of Thorns Conference 1980, Cambridge University Press, Cambridge, 1981, pp. 119-127.

[9] S. G. Hartke, P. R. J. Östergård, D. Bryant, and S. I. El-Zanati, The nonexistence of a $\left(K_{6}-e\right)$-decomposition of the complete graph $K_{29}$, J. Combin. Des. 18 (2010), 94-104.

[10] P. Kaski and P. R. J. Östergård, There are exactly five biplanes with $k=11$, J. Combin. Des. 16 (2008), 117-127.

[11] P. Kaski and P. R. J. Östergård, Classification Algorithms for Codes and Designs, Springer, Berlin, 2006.

[12] P. Kaski and O. Pottonen, libexact user's guide, version 1.0, HIIT Technical Reports 2008-1, Helsinki Institute for Information Technology HIIT, 2008.

[13] D. E. Knuth, Dancing links, in: J. Davies, B. Roscoe, and J. Woodcock (Eds.), Millennial Perspectives in Computer Science, Palgrave, Houndmills, 2000, pp. 187-214.

[14] C. W. H. Lam, How reliable is a computer-based proof?, Math. Intelligencer 12 (1990), no. 1, 8-12.

[15] J. H. van Lint, On ovals in PG(2,4) and the McLaughlin graph, in: P. J. de Doelder, J. de Graaf, and J. H. van Lint (Eds.), Papers Dedicated to J. J. Seidel, Report 84-WSK-03, Department of Mathematics and Computing Science, Eindhoven University of Technology, 1984, pp. 234-255. 
[16] S. Linton, Finding the smallest image of a set, in: ISSAC '04: Proceedings of the 2004 International Symposium on Symbolic and Algebraic Computation, ACM, New York, 2004, pp. 229-234.

[17] R. Mathon, Searching for spreads and packings, in: J. W. P. Hirschfeld, S. S. Magliveras, and M. J. de Resmini (Eds.), Geometry, Combinatorial Designs and Related Structures, Cambridge University Press, Cambridge, 1997, pp. 161-176.

[18] B. D. McKay and A. Piperno, Practical graph isomorphism, II, J. Symbolic Comput. 60 (2013), 94-112.

[19] J. McLaughlin, A simple group of order 898,128,000, in: R. Brauer and C.-H. Sah (Eds.), Theory of Finite Groups, Benjamin, New York, 1969, pp. 109-111.

[20] S. Niskanen and P. R. J. Östergård, Cliquer user's guide, version 1.0, Technical Report T48, Communications Laboratory, Helsinki University of Technology, Espoo, 2003.

[21] C. Pech and S. Reichard, Enumerating set orbits, in: M. Klin, G. A. Jones, A. Jurišić, M. Muzychuk, and I. Ponomarenko (Eds.), Algorithmic Algebraic Combinatorics and Gröbner Bases, Springer, Berlin, 2009, pp. 137-150.

[22] S. Reichard, An algorithm for the construction of partial geometries with given point graphs, Technical Report MATH-AL-12-1997, Technische Universität Dresden, 1997.

[23] S. Reichard, personal communication.

[24] D. J. Roy, Confirmation of the Non-existence of a Projective Plane of Order 10, MSc Thesis, Carleton University, Ottawa, 2010.

[25] L. H. Soicher, Is there a McLaughlin geometry?, J. Algebra 300 (2006), 248-255. Logfile of computations:

http://www.maths.qmul.ac.uk/ 〜leonard/mclgeom.txt

[26] L. H. Soicher, The GRAPE package for GAP, Version 4.6.1, 2012, http://www.maths.qmul.ac.uk/ leonard/grape/

[27] J. A. Thas, Partial geometries, in: C. J. Colbourn and J. H. Dinitz (Eds.), Handbook of Combinatorial Designs, 2nd ed., Chapman \& Hall/CRC, Boca Raton, 2007, pp. 557-561. 